\title{
The finer scale of consciousness: quantum theory
}

\author{
Tianwen Li ${ }^{1 \#}$, Hailiang Tang ${ }^{1 \#}$, Jianhong Zhu ${ }^{1}$, John H. Zhang ${ }^{2}$ \\ ${ }^{1}$ Department of Neurosurgery, Fudan University Huashan Hospital, National Key Laboratory of Medical Neurobiology, the Institutes of Brain \\ Science and the Collaborative Innovation Center for Brain Science, Shanghai Medical College, Fudan University, Shanghai 200040, China; ${ }^{2}$ Center \\ for Neuroscience Research, Loma Linda University School of Medicine, Loma Linda, CA, USA \\ Contributions: (I) Conception and design: T Li, J Zhu, H Tang; (II) Administrative support: J Zhu, JH Zhang; (III) Provision of study materials or \\ patients: T Li, H Tang; (IV) Collection and assembly of data: T Li, JH Zhang; (V) Data analysis and interpretation: All authors; (VI) Manuscript \\ writing: All authors; (VII) Final approval of manuscript: All authors. \\ "These authors contributed equally to this work. \\ Correspondence to: Jianhong Zhu. No.12 Urumqi Mid Road, Shanghai 200040, China. Email: jzhu@fudan.edu.cn; John H. Zhang. Director, Center \\ for Neuroscience Research, Loma Linda University School of Medicine, 11175 Campus St, Loma Linda, CA 92350, USA. \\ Email: johnzhang3910@yahoo.com.
}

\begin{abstract}
Consciousness is a multidisciplinary problem that has puzzled all human beings since the origin of human life. Being defined in various pointcuts by philosophers, biologists, physicists, and neuroscientists, the definitive explanation of consciousness is still suspending. The nature of consciousness has taken great evolution by centering on the behavioral and neuronal correlates of perception and cognition, for example, the theory of Neural Correlates of Consciousness, the Global Workspace Theory, the Integrated Information Theory. While tremendous progress has been achieved, they are not enough if we are to understand even basic facts-how and where does the consciousness emerge. The Quantum mechanics, a thriving branch of physics, has an inseparable relationship with consciousness (e.g., observer effect) since Planck created this subject and its derived quantum consciousness theory can perfectly fill this gap. In this review, we briefly introduce some consciousness hypotheses derived from quantum mechanics and focus on the framework of orchestrated objective reduction (Orch-OR), including its principal points and practicality.
\end{abstract}

Keywords: Consciousness; quantum mechanics; uncertainty principle; orchestrated objective reduction theory (Orch-OR theory); microtubule

Submitted Aug 14, 2019. Accepted for publication Aug 23, 2019.

doi: 10.21037/atm.2019.09.09

View this article at: http://dx.doi.org/10.21037/atm.2019.09.09

\section{Introduction}

The nature of consciousness, once the exclusive realm of philosophers, has been gradually penetrated by neuroscientists, biologists, and physicists. Consciousness has always been defined as the Hard Problem in these subjects (1-4). With the emergence of unprecedented devices and the development of multidisciplinary experiments in different research fields, more details of this hard problem have been revealed, especially in quantum mechanics and neuroscientific fields. Since we decided to dive into the ocean of consciousness, many may think that the "water molecule" is our best beginning to disentangle this multidisciplinary conundrum. Thus, various interpretations of consciousness originated at the neuronal and molecular levels. The past 30 years have witnessed the tremendous and remarkable achievements of the neural correlates of consciousness $(5,6)$. Nevertheless, there are many riddles that these "neuronal and molecular" models cannot explain (7). As a supplement to the theory of macroscopic physics (classical Newton's mechanics), quantum mechanics has existed only for approximately one hundred years; however, some quantum mechanics terminologies have been ever prevalent, e.g., quantum entanglement, quantum coherence, wave-function collapse, and the most famous 
cat worldwide-Schrödinger's cat. Quantum computers are considered the brightest new star in the quantum field and increasingly fascinate quantum physicists and information technology specialists. Advances in new materials and cryogenic physics have led to remarkable breakthroughs in quantum computing in recent years. Large commercial quantum computer systems that were invented by IBM (8-10) or the University of Science and Technology of China (11-13) could soon be operational within five years. Because quantum mechanics deals with the tiniest constituents of the material world, it seems capable of elucidating numerous unsolved and tough problems. Quantum theory, a branch from the finer scale of consciousness, has been accompanied by numerous controversies since its inception, but abundant proof demonstrated that this theoretical framework is capable of explaining the majority of consciousness problems that traditional neuroscience could not, especially the orchestrated objective reduction (Orch-OR) theory introduced by Penrose and Hameroff.

\section{Origination}

Consciousness was brought onto the scene that was ablaze with lights for more than 2,500 years since Socrates postulated that the cerebrum created consciousness, while Aristotle argued that mental qualities belonged to fundamental reality. More recently, Descartes proposed "Cogito ergo sum" in the Late Renaissance (14), and its definition and biological basis remain impasses such that no ultimate version has been reached, although there are various explanations based on its different aspects. Undoubtedly, any objective definition trying to explain this subjective problem seems feeble, which accounts for its official position-a hard problem. Compared with consciousness, quantum mechanics that was first invented approximately one hundred years ago is much younger. However, the latecomers have the upper hand. The last 100 years have witnessed the rapid development of quantum mechanics, and various significant breakthroughs have been accomplished by quantum physicists, while evolution in consciousness has progressed at a snail's pace.

\section{Initial exploration}

In the 1930s, the Hungarian physicist Eugene Wigner considered it logical that the quantum description of an object is influenced by the mind that comes into our consciousness. In logic, solipsism may be consistent with current quantum mechanics (15). From the theory of the brain quantum field, Hiroomi Umezawa interpreted memory and other conscious phenomena as the result of the energy exchange of energy particles in the cerebral cortical field (16-19). Before long, Herbert Fröhlich proposed that the quantum field in the cell membrane condensed into the same state with quantum coherence during charge oscillation, namely, the Bose-Einstein condensate $(20,21)$. Next, following Fröhlich, Marshall introduced that BoseEinstein condensate produced from pumped phonons was the candidate for processing consciousness (22). John Wheeler, a noble American physicist, even suggested that the existence of life, including all life with "observation" ability, may have transformed many of the possible "quantum pasts" into real history. From this perspective, we have been participants since the beginning of the universe. Stunningly but perceptibly, we live in a "participating universe" (23). In the book of Quantum Theory (24), Bohm proposed that implicate order could apply to both the material world and consciousness, which was capable of explaining the relationship between them. In his opinion, mind and matter are projections into the explicate order from the underlying implicate order. By comparing optical holography with visual memory, Gabor attempted to explain the connection between human temporal recall and quantum mechanics (25). Stapp postulated a global collapse, a 'mind like' wave-function collapse that exploited certain aspects of the quantum Zeno effect within the synapses $(26,27)$, distinct from the idea of Hameroff and Penrose (described in the "OrchOR theory" section). Although his theory was based on synapses, a junction structure between neurons within the brain, unfortunately, he did not integrate his exhaustive quantum mechanical theory into an optimal interpretation with neurological or neuronal substrates. Jibu and Yasue believed that the movement of corticon and boson in the dipolar vibrational fields distributed along protein filaments of the cytoskeleton and extracellular matrices and surrounding water fields of the brain was the process of quantum information transmission, which ultimately led to the formation of quantum coherent states in the cortex (28-33). Disagreements and rebuttals were inevitable due to these ideas, and theories were simply overflowing from those physicists' minds, lacking factual neuroanatomical evidence and even systematic contexture (34-36). 


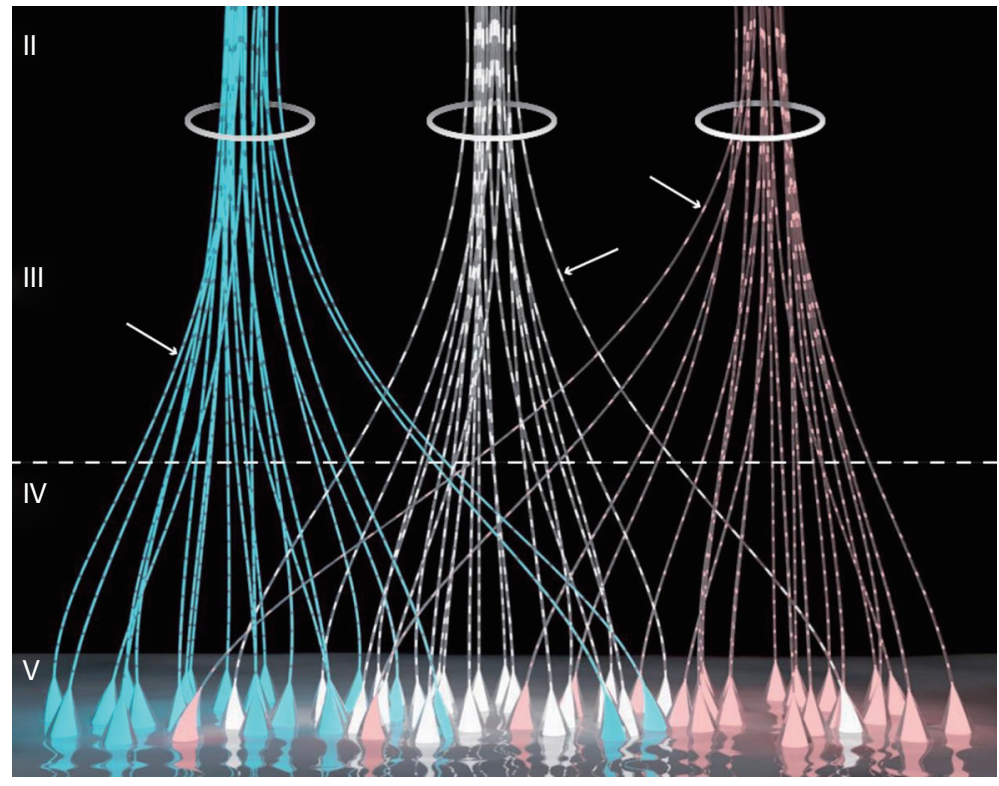

Figure 1 Schematic diagram of dendrons and psychons of layer $\mathrm{V}$ pyramidal cells. Each triangle represents a pyramidal cell, and the ascending lines extending from the top of triangles are apical dendrites. Light spots (arrowhead), which are transported along apical dendrites with different colors, represent different kinds of psychons that could give rise to unique experiences. The dendrites cluster automatically while ascending to the superficial layer of cortex to configure dendrons (circle) according to the different psychons they transmit. Additionally, pyramidal cells are divided into different groups based on the distinct conscious experiences that they process (37).

\section{Gradual systematization}

Many biological scientists have tried to find a quantum-level substance that can be transmitted between neurons, such as a neurotransmitter, which conveys consciousness-related information (37). Based on "consciousness evolutionism", which emphasized that it is unlikely that consciousness came to higher creatures as a sudden leap, a finer world had been built. In this microgranular mental world, the "quantumlevel substance" is the mental microunits called psychons, wandering around the mammalian neocortex (Figure 1). Each kind of psychon represents a unitary experience of consciousness-qualia. Like conventional neurotransmitters, psychons are transported along dendrons formed by apical dendrites (also their branches) of pyramidal cells in layers V, III, and II. During the period in which synapse structure was discovered, its foundational hypothesis had no reason to deviate from synaptic micro-property.

Following the analogy of probabilistic fields of quantum mechanics and neural events arising from mental activity (38), as well as the hypothesis of dendrons and synaptic structure mentioned above, a more complete and systematic framework was constructed by Beck (37). More quantum mechanics mechanisms were also introduced. Exocytosis, as an intriguing discovery, was chosen as the indispensable and pivotal step. In the standby phase of the synaptic bouton, exocytosis was seen as a staged quantal event based on the quantum tunneling effect, resulting in momentarily increasing the probability of exocytosis (actually, it was named the selection of events in quantum mechanics). This selection mechanism augmented the likelihood for exocytosis and led to amplified excitatory postsynaptic potentials. Then, a coherent coupling of boutons in a dendron occurred. Because the dendrons in the cerebral cortex contain countless synaptic connections, there is an enormous amount of quantum transmission in the human brain, which inevitably leads to the randomness and freedom of mental phenomena-free will.

However, after more than twenty years, no subsequent development of this ancestral hypothesis occurred; additionally, the true identity of psychons was not discovered, despite countless works focused on cortical architecture (39). Various limitations persisted in these theories; however, they probably marked the beginning of the convergence of neuroscience and quantum mechanics 


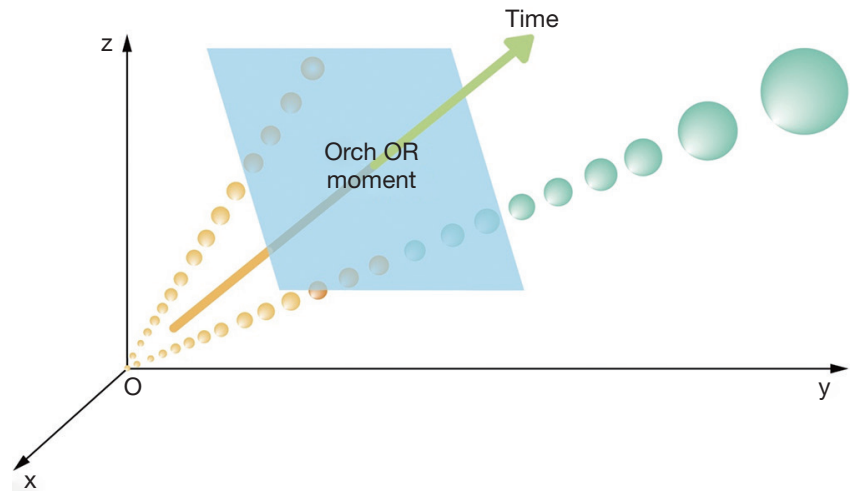

Figure 2 Conceptual graph of quantum space-time structure. The quantum particle vibrates from the start point $O$. Based on the uncertainty principle, the superposed condition of the particle determines that there are two possible tracks of particle progress in 3-dimensional xyz space. The orange and green large arrow indicates time, the fourth dimension. The initial variance between the two tracks is tiny ( $\mathrm{O}$ point), but over time, the variance increases. One of its possible tracks would disappear after reaching the threshold at the moment of Orch-OR, leaving the other as certain and valid. The blue panel is the conscious moment of Orch-OR (44).

for further understanding of consciousness.

\section{Orch-OR theory}

It was widely accepted that most neuronal communication and information transmission initially occurred on receptors and ligands (especially among synapses in the central nervous system) on the cell membrane, followed by second messengers that broadcast or transfer the information to various parts of the interior cell. Almost all basic studies in neurobiology converge on the various receptors, ligands and signaling pathways. However, are we $100 \%$ certain about this prerequisite basis of neuroscience? Rather than the conventional receptors and ligands of the membrane, the principal cellular components of the Orch-OR theory are microtubules that are mostly considered pivotal structures for material transportation, cell movement, mitosis and establishment and maintenance of cell form and function.

To date, this theory has remained one of the most acceptable and continuous theories that covers in detail quantum physics, quantum gravity, quantum information theory, molecular biology, neuroscience, cognitive science, philosophy, and anesthesiology. Additionally, this theory was known to neurobiologists who were interested in the "Hard Problem" as well as physicists and philosophers.

Under the background of rapid development of world computer technology, Hameroff likened the flow of information in the brain to computers in which microtubules were to the brain what transistors were to the computer (40-43). Inspired by this fantastic analogy and Gödel's incompleteness theorems, in The Emperor's New Mind (44) published in 1989, Roger Penrose first attached the quantum effect in human cognition. For example, he considered whether consciousness can affect quantum mechanics or vice versa and that quantum mechanics itself might be included in consciousness. Penrose suggested that the "objective collapse", that is, the collapse and superposition of quantum interference, is a real physical process, similar to the bursting of bubbles (44). Furthermore, consciousness was the product of quantum space-time structure (Figure 2), which was inextricably related to the universe, and the theory describing the relationship between consciousness and the universe was the Orch-OR theory (44). These quantum theories facilitated the emergence of later biological hypotheses of consciousness based on quantum mechanics.

Originally, the cytoskeleton was proposed as the cell's nervous system and biological controller (nanocomputer), which had self-stabilizing logic algorithms introduced by Hameroff who was inspired by the subtle link between Fröhlich's coherent excitations and tubulin subunits in microtubules $(40,42,45-47)$. He invoked that microtubules could be the fundamental units involving information processing in our enigmatic brain (48), e.g., visual identity $(49,50)$, learning (42), cognition (42), and memory (49,51). An unprecedented collaboration heralded the advent of the Orch-OR theory. Illuminated by the structure of microtubules, Penrose posited that the microtubules composed of protein polymers played an essential role in the understanding of human consciousness from the perspective of quantum mechanics (52). Co-established by Hameroff and Penrose, the Orch-OR theory approached consciousness from various aspects and became richer and more profound with new discoveries in neuroscience and development of research methods and instruments.

Microtubules and tubulins, as the elaborate and dynamic three-dimensional network, are found in almost all eukaryotes $(53,54)$ and some prokaryotes $(55-57)$. Microtubules are stiff noncovalent polymers of $\alpha$ - and $\beta$-tubulin functioning as the cell cytoskeleton (58), the spindle during mitosis (59) and 


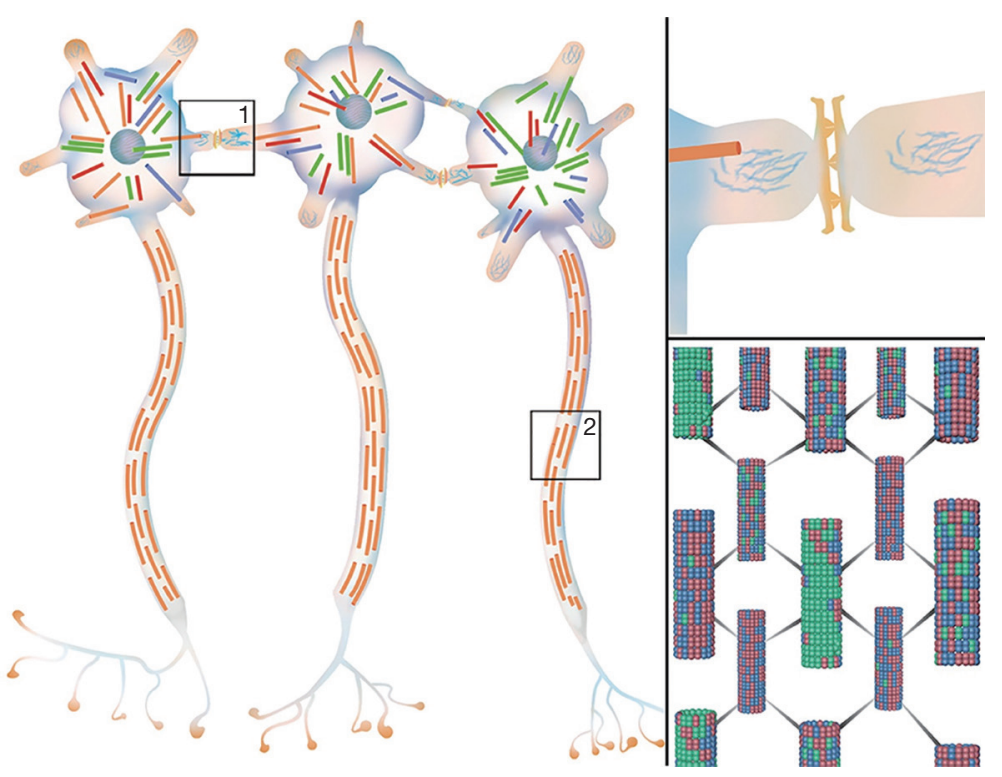

Figure 3 Neurons with gap junctions and microtubules. Left: schematic of the morphology of adjacent neurons. Microtubules in the soma and dendrites are clustered randomly and multi-oriented, while their counterparts in axons stretch from the axon hillock to the terminal in the same direction. Upper right: the magnified version of box 1 in the left graph. The gap junctions link nearby dendrites of different neurons, which make instantaneous interneuronal communication possible. Lower right: the magnified version of box 2 in the left graph. Microtubules in axons are regularly arranged in the same direction interconnected by microtubule-associated proteins (69).

axonemes of cilia and flagella, playing dispensable roles in cell support, migration $(60,61)$, development (62-64), gene regulation $(65,66)$ and axoplasmic transport $(67)$. Because of the particularity of neurons, e.g., nondividing properties and electrical signal conduction, microtubules have many specific characteristics. There are many more tubulins for microtubules within neurons than within somatic cells. The nondividing property determines its unique stability. Furthermore, each part of microtubules within neurons is also distinct. Microtubules in axons extend with the same orientation from the centrosome near the nucleus to the axon terminal. In contrast, microtubules in dendrites and cell bodies are disordered and multi-oriented $(68,69)$ (Figure 3). The caps on dendritic and somatic microtubules could prevent treadmilling-like depolymerization that occurred in other somatic cells, which accounts for the steady and probable basis of information processing (70,71). Microtubule-associated proteins (MAPs), actin, and intermediate filaments are the key structures precipitating proper microtubule function (Figure 3). Microtubules are interconnected by MAPs; thus, their physiological activities are modulated by MAPs in different phosphorylation states (72). One of the most famous MAPs is the tau protein, which has been widely studied in Alzheimer's disease (73).
Tubulins were deconstructed long ago. The study of tubulin amino acids was more advanced than the study of their $\alpha$ and $\beta$ functional groups $(74,75)$. In the Orch-OR theory, one of the most creative and prerequisite points is that tubulins are in a quantum superposition state according to the Heisenberg uncertainty principle; similar to qubits in quantum mechanics, they are 1 and 0 instead of 1 or 0 in computer computation.

Similar to the initiation of information transmission between neuronal synapses in traditional neurobiology, after the neurotransmitter binds to the receptor on the postsynaptic membrane, the activation of ion channel type receptors increases the intracellular calcium ion concentration. Alternatively, binding to a metabolic receptor activates a second messenger and subsequent signaling pathways. Conformational changes, e.g., phosphorylation and dephosphorylation, occur in microtubules and MAPs due to changes in calcium concentration and activation of various signaling pathways $(71,76)$. These subtle changes caused by neurotransmitters from synaptic inputs could "orchestrate" tubulin states controlled by dipole couplings (one kind of intermolecular force), thus leading to microtubule simulation $(50,77)$ (Figure 4). Tubulin quantum coherent superpositions and computations are increasingly 


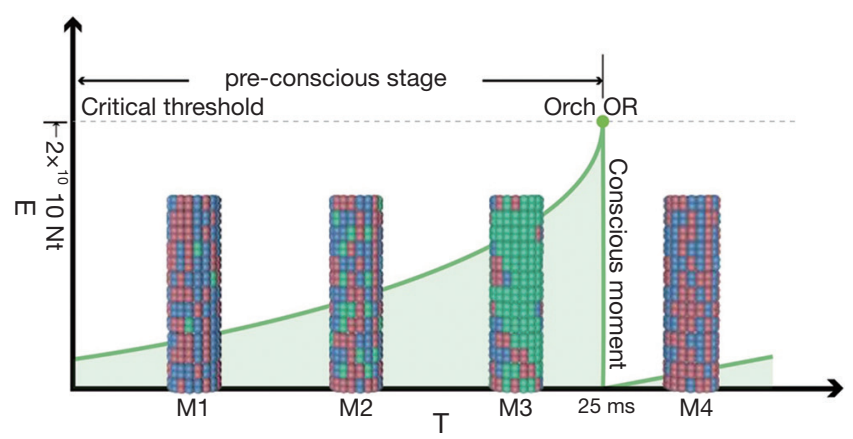

Figure 4 Orch-OR event. Minor tubulins begin with classical computing (green tubulins in M1), which leads to quantum coherent superposition and quantum computing (expanding of green tubulins in M2 and M3). When the critical threshold of coherence to quantum gravity is met, Orch-OR will occur; thus, the entire condition of the microtubule returns to the original pattern (M4). The conscious Orch-OR event occurs in the M3 to M4 transition. The area under the curve represents superposed mass energy $\mathrm{E}$ with self-collapse time $\mathrm{T}$, which is consistent with the formula of $\mathrm{E}=\hbar / \mathrm{T}$. $\mathrm{E}$ may be described as $\mathrm{Nt}$, the number of tubulins whose mass separation (and separation of underlying space time) for time $\mathrm{T}$ will self-collapse. Hameroff posited that $\mathrm{T}=25 \mathrm{~ms}$ (e.g., $40 \mathrm{~Hz}$ oscillations), $\mathrm{Nt}=2 \times 10^{10}$ tubulins $(69,78)$. M1, microtubule state $1 ; \mathrm{M} 2$, microtubule state 2; M3, microtubule state $3 ; M 4$, microtubule state 4 .

combined to augment their superposed mass energy. Once the energy meets the critical threshold of quantum gravity, self-collapse occurs. That is, at this moment, the consciousness event occurs (according to Hameroff and Penrose, consciousness is discrete and independent rather than continuous) (Figure 4). Notably, under the "warm and noisy" brain, the information flow of neurotransmitters could prevent random environmental decoherence from overwhelming unitary evolution (derived from Schrödinger equation) quantum procedures. The whole orchestration and self-collapse process could be represented by a function schematic graph (Figure 4). From the equation $\mathrm{E}=\hbar / \mathrm{T}$ ( $\hbar$ is Planck's constant divided by $2 \varpi, T$ is time, $\mathrm{E}$ is the energy level that could be represented by the number of tubulins (Nt) in the Orch-OR theory), we could infer that only when an animal possesses enough microtubules (or a large enough brain) could it give rise to consciousness in a relatively short and realizable period of time. In the modern physical field, it was suggested that reality is composed of 3-dimensional space and 1-dimensional time. As Figure 2 shows, from the beginning, particulates (microtubules) exist two alternative states in 3-dimensional space (just like Schrödinger's cat in the uncertainty principle). Additionally, with the orchestration of synaptic inputs over time, once the threshold is met, objective reduction occurs, and their state is confirmed $(69,79)$. Therefore, only one certain state continued, while the other disappeared. A neuron could accept numerous distinct neurotransmitters by its dendrites and soma, that is, there must be integration between different dendritic and somatic microtubules that precipitates dendritic and somatic microtubules within a neuron to meet this critical point congruously in case their quantum coherence and computation are chaotic. Gap junctions, which are not of much concern in ordinary neuroscience research, are considered to play an important role in quantum tunneling among dendrites in the macroscopic quantum coherent state, information exchange and mutual adjustment between neurons (49,80-82) (Figure 3). Then, the axon senses the instantaneous conscious events and fires to convey outputs to control advanced life intelligence activities and behaviors. In conclusion, dendritic and somatic microtubules' tubulins are initially in superposed states. Synaptic inputs orchestrate these tubulins such that their state tends to be unified, and total energy increases. Then, the threshold is met, followed by Orch-OR and a conscious event. Finally, all the tubulins and microtubes return to their original state, ready to accept the next synaptic input (Figure 4).

However, debates have become increasingly turbulent since the introduction of the Orch-OR theory. Some scientists, including philosophers, physicists and neuroscientists, support that their work heralded a new level of understanding of consciousness and was worthy of further development and experimentation (83-88). However, numerous rebuttals have emerged since the inception of the Orch-OR theory. These criticisms covered biological, gravitational and quantum fields to which Hameroff and Penrose responded one by one with detailed biological evidence, quantum mechanical equations and theoretical frameworks in "Consciousness in the universe: A review of the 'Orch OR' theory" (69). However, this review generated an even larger wave of criticism. Comments sprang up like mushrooms $(84,89,90)$. One of the most noted retorts was proposed by Baars (89) in his article "Consciousness, biology and quantum hypotheses", which caused this debate to reach the climax. Baars invoked some actual questions that many other critics mentioned. (I) All plant and animal cells are composed of microtubules, and some of their MAPs are similar. (II) Countless previous studies have proven that the cerebral cortex and thalamus 
are strongly implicated in conscious experience, while on a quantum basis, their specificity did not emerge. (III) More than half of the human brain region, especially the cerebellum, does not hold consciousness content; however, neurons in these regions also have identical microtubules. (IV) There is no known quantum-level proof to account for the difference between conscious versus unconscious brain events. (V) Consciousness must evolve with biological evolution. Hameroff replied with reasonable explanations that validated Orch-OR as an appropriate and qualified theory to surmise consciousness, despite the increasingly detailed development that should be interpreted (91). An inevitable situation was that arguments about Baars's direct exclusion of "freshman" quantum theory burst into more debates in which critics stated that Baars should be more generous regarding quantum consciousness theory due to its practical basis, which could enrich our understanding of consciousness (92-95). In addition, more theories and rebuttals could bring more attention and science funding into the "puzzle game" of consciousness that has confused and captivated philosophers, neuroscientists, and physicists for ages.

\section{Conclusion and perspective}

Quantum mechanics has been inextricably linked with consciousness since its birth. Various proposals and theories have been put forward to lift the veil of consciousness. In this review, we summarized the main quantum theory of consciousness of which the theories based on neural structure were the principal parts. Undoubtedly, the Orch-OR theory co-established by theoretical physicist Penrose and neuroscientist Hameroff is currently the most convincing theory. Even more exciting, with the emergence of new drugs, new research methods, and new quantum technologies, this theory is constantly being enriched and perfected. Especially in the research of anesthesiology (96-100), memory (71), cognition (42,101-103), neural synchrony (104) and vision (49), mounting results and evidence indicated the Orch-OR theory could be selfexplanatory and could be invoked to many different conscious backgrounds. More recently, Li et al. found that xenon's (one kind of anesthetic) nuclear spin could impair its own anesthetic power, which involves a neural quantum process (105). Thus, the quantum theory of consciousness is increasingly gaining more supporters. With the dedication of these supporters, the quantum theory of consciousness will be gradually completed and will be able to explain the hard problem systematically and comprehensively. As the enigmatic riddle of consciousness has remained intractable, we need more theories and hypotheses to attract enough attention and maintain lively debate. This conflict is the only way for human beings to explore the truth. Since there is no conclusive scientific mechanism of consciousness, as one of the most systemic and convincing theories among various theories of consciousness, the Orch-OR theory deserves our deeper understanding and study. Let us not put Descartes before the horse (94). It is possible to disperse the fog of our ignorance and shed light on new knowledge regarding consciousness.

\section{Acknowledgments}

We thank Yong Chen, School of Arts and Media, Tongji University for figure support.

Funding: This study was funded by Ministry of Science and Technology of China (2018YFA0107900), The National Nature Science Foundation (No. 31771491), and Shanghai Municipal Government (2019CXJQ01).

\section{Footnote}

Conflicts of Interest: The authors have no conflicts of interest to declare.

Ethical Statement: The authors are accountable for all aspects of the work in ensuring that questions related to the accuracy or integrity of any part of the work are appropriately investigated and resolved.

\section{References}

1. Kuehni RG. Consciousness Explained, by Daniel C. Dennett, Little, Brown, Boston, MA, 1991, xiii + 511 pp., Color Res Appl 2010;17:295-7.

2. MarcelKinsbourne. Time and the observer: The where and when of consciousness in the brain. Behav Brain Sci 1992;15:183-201.

3. Koch C, Crick F. The zombie within. Nature 2001;411:893.

4. Wegner DM. The Illusion of Conscious Will. Mit Pr 2003;159:197-213.

5. Crick F, Koch C. Some Reflections on Visual Awareness. Cold Spring Harb Symp Quant Biol 1990;55:953.

6. Koch C, Massimini M, Boly M, et al. Neural correlates of consciousness: Progress and problems. Nat Rev Neurosci 
2016;17:307-21.

7. Brancucci A, Lugli V, Perrucci MG, et al. A frontal but not parietal neural correlate of auditory consciousness. Brain Struct Funct 2016;221:463-72.

8. Alvarez-Rodriguez U, Sanz M, Lamata L, et al. Quantum Artificial Life in an IBM Quantum Computer. Sci Rep 2018 4;8:14793.

9. Bravyi S, Gambetta JM, Mezzacapo A, et al. Tapering off qubits to simulate fermionic Hamiltonians. Quantum Physics 2017, arXiv:1701.08213.

10. Bravyi S, Gosset D, König R. Quantum advantage with shallow circuits. Science 2018;362:308-311.

11. Wang XL, Cai XD, Su ZE, et al. Quantum teleportation of multiple degrees of freedom of a single photon. Nature 2015;518:516.

12. Ding X, He Y, Duan ZC, et al. On-Demand Single Photons with High Extraction Efficiency and Near-Unity Indistinguishability from a Resonantly Driven Quantum Dot in a Micropillar. Phys Rev Lett 2016;116:020401.

13. Chen MC, Wu D, Su ZE, et al. Efficient Measurement of Multiparticle Entanglement with Embedding Quantum Simulator. Phys Rev Lett 2016;116:070502.

14. Sordillo P. Cogito Ergo Sum. Ital Am 2005;23:73.

15. Guth E. Gruppentheorie und ihre Anwendung auf die Quantenmechanik der Atomspektren. Monatshefte Für Math Und Phys 1932;39:A51.

16. Ricciardi LM, Umezawa H. Brain and physics of manybody problems. Kybernetik 1967;4:44-8.

17. Stuart CI, Takahashi Y, Umezawa H. On the stability and non-local properties of memory. J Theor Biol 1978;71:605-18.

18. Stuart CI, Takahashi Y, Umezawa H. Mixed-system brain dynamics: Neural memory as a macroscopic ordered state. Found Phys 1979;9:301-27.

19. Roy S, Kafatos M. Quantum processes and functional geometry: new perspectives in brain dynamics. Forma 2004;1:69-84.

20. Fröhlich H. Long-range coherence and energy storage in biological systems. Int J Quantum Chem 1968;2:641-9.

21. Chapin FS. Week's Citation. 1988;1989.

22. Marshall IN. Consciousness and Bose-Einstein condensates. New Ideas Psychol 1989;7:73-83.

23. Wheeler JA. Include the Observer in the Wave Function? In: Lopes JL, Paty M. editors. Quantum Mechanics, A Half Century Later. Episteme (A Series in the Foundational, Methodological, Philosophical, Psychological, Sociological and Political Aspects of the Sciences, Pure and Applied),
1977 vol 5. Springer, Dordrecht.

24. Bohm D. Quantum theory. Phys Today 1952;5:23-4.

25. Gabor D. Holographic Model of Temporal Recall. Nature 1968;217:584.

26. Stapp HP. Mind, matter, and quantum mechanics. Found Phys 1982;12:363-99.

27. Mahler G. Mind, Matter and Quantum Mechanics (2nd edition). J Phys A Gen Phys 2004;37:6857.

28. Jibu M, Yasue K. Quantum Measurement by Quantum Brain. 1995.

29. Jibu M, Yasue K. A physical picture of Umezawa's quantum brain dynamics. Cybern Syst Res 1992;92:797-804.

30. Pribram KH. Rethinking Neural Networks: Quantum Fields and Biological Data. Taylor \& Francis, 2014. Available online: https://books.google.com/ books?id=L7NQAwAAQBAJ

31. Jibu M, Yasue K. Intracellular quantum signal transfer in Umezawa's quantum brain dynamics. Cybern Syst 1993;24:1-7.

32. Jibu $M$, Yasue $\mathrm{K}$. Introduction to quantum brain dynamics in, Nature, Cognition and System ed, Carvallo, M. Kluwer Academic, 1993.

33. Jibu M, Yasue K. Quantum Brain Dynamics and Consciousness: An introduction. John Benjamins Publishing Company, 1995. Available online: https:// books.google.com/books?id=elh1MqqymRwC

34. Stenger V. The myth of quantum consciousness. Humanist 1992;53:13-5.

35. Chalmers DJ. Facing Up to the Problem of Consciousness. J Conscious Stud 1994;2:200-19.

36. Gibson KR. Book Review: The Conscious Mind: In Search of a Fundamental Theory. David J. Chalmers. Int J Quantum Chem 2015;66:107-8.

37. Beck F, Eccles JC. Quantum aspects of brain activity and the role of consciousness. Proc Natl Acad Sci U S A 1992;89:11357-61.

38. Eccles J C. Do mental events cause neural events analogously to the probability fields of quantum mechanics? Proc R Soc Lond B Biol Sci 1986;227:411-28.

39. Amunts K, Zilles K. Architectonic Mapping of the Human Brain beyond Brodmann. Neuron 2015;88:1086-107.

40. Hameroff SR, Watt RC. Information processing in microtubules. J Theor Biol 1982;98:549-61.

41. Smith SA, Watt RC, Hameroff SR. Cellular automata in cytoskeletal lattices. Phys D Nonlinear Phenom 1984;10:168-74.

42. Rasmussen S, Karampurwala H, Vaidyanath R, et al. 
Computational connectionism within neurons: A model of cytoskeletal automata subserving neural networks. Phys D Nonlinear Phenom 1990;42:428-49.

43. Rasmussen S, Karampurwala H, Vaidyanath R, et al. Emergent computation in microtubule model networks. In: Century, International Conference of the IEEE Engineering, 2002.

44. Penrose R. The Emperor's New Mind. Neuroscience 1991;37:285-6.

45. Koruga D. Ultimate computing: Biomolecular consciousness and nanotechnology. by Stuart R. Hameroff, Elsevier Science Publishers B.V. Amsterdam, The Netherlands, 1987:357. illus. ISBN 0-444-70283-0. Biosystems 1987;22:83-4.

46. Hameroff SR, Rasmussen S. Information Processing in Microtubules: Biomolecular Automata and Nanocomputers. 1989.

47. Lahoz-Beltra R, Hameroff SR, Dayhoff JE. Cytoskeletal logic: a model for molecular computation via Boolean operations in microtubules and microtubule-associated proteins. Biosystems 1993;29:1-23.

48. Jibu M, Hagan S, Hamerof SR, et al. Quantum optical coherence in cytoske1etal microtubules: implications for brain function. Biosystems 1994;32:195-209.

49. Woolf NJ, Hameroff SR. A quantum approach to visual consciousness. Trends Cogn Sci 2001;5:472-8.

50. Hameroff SR. 'Funda-Mentality': Is the conscious mind subtly linked to a basic level of the universe? Trends Cogn Sci 1998;2:119-24.

51. Hameroff SR, Craddock TJ, Tuszynski JA. "Memory bytes" - molecular match for CaMKII phosphorylation encoding of microtubule lattices. J Integr Neurosci 2010;9:253-67.

52. Penrose BR. Shadows of the Mind. Oxford Univ Press, Oxford 1996;43:17-24.

53. Carlier M F, Pantaloni D. Kinetic analysis of guanosine 5'-triphosphate hydrolysis associated with tubulin polymerization. Biochemistry 1981;20:1918.

54. Desai A, Mitchison TJ. Microtubule polymerization dynamics. Annu Rev Cell Dev Biol 1997;13:83-117.

55. Margolin W. FtsZ and the division of prokaryotic cells and organelles. Nat Rev Mol Cell Biol 2005;6:862-71.

56. Pilhofer M, Ladinsky MS, Mcdowall AW, et al.

Microtubules in bacteria: Ancient tubulins build a fiveprotofilament homolog of the eukaryotic cytoskeleton. Plos Biol 2011;9:e1001213.

57. Schlieper D, Oliva MA, Andreu JM, et al. Structure of bacterial tubulin BtubA/B: evidence for horizontal gene transfer. Proc Natl Acad Sci U S A 2005;102:9170-5.

58. Kueh HY, Mitchison TJ. Structural plasticity in actin and tubulin polymer dynamics. Science 2009;325:960-3.

59. Mitchison T, Kirschner M. Dynamic instability of microtubule growth. Nature 1984;312:237-42.

60. Mikhailov A, Gundersen GG. Relationship between microtubule dynamics and lamellipodium formation revealed by direct imaging of microtubules in cells treated with nocodazole or taxol. Cell Motil Cytoskeleton 1998;41:325-40.

61. Waterman-Storer CM, Worthylake RA, Liu BP, et al. Microtubule growth activates Rac1 to promote lamellipodial protrusion in fibroblasts. Nat Cell Biol 1999; $1: 45-50$

62. van Eeden F, St Johnston D. The polarisation of the anterior-posterior and dorsal-ventral axes during Drosophila oogenesis. Curr Opin Genet Dev 1999;9:396-404.

63. Beddington RS, Robertson EJ. Axis development and early asymmetry in mammals. Cell 1999;96:195-209.

64. Tucker RP. The roles of microtubule-associated proteins in brain morphogenesis: a review. Brain Res Brain Res Rev 1990;15:101-20.

65. Christian O, Dominika I, Angela G, et al. Modulation of the expression of connective tissue growth factor by alterations of the cytoskeleton. J Biol Chem 2010;85:A42.

66. Rosette C, Karin M. Cytoskeletal control of gene expression: depolymerization of microtubules activates NF-kappa B. J Cell Biol 1995;128:1111-9.

67. Hirokawa N, Noda Y, Tanaka Y, et al. Kinesin superfamily motor proteins and intracellular transport. Nat Rev Mol Cell Biol 2009;10:682-96.

68. Böhm KJ. P. Dustin, Microtubules (Second Totally Revised Edition). XV + 482 S., 175 Abb. Berlin-Heidelberg-New York-Tokyo 1984. Springer-Verlag. ISBN: 3-540-13283-X. J Basic Microbiol 2010;25:630.

69. Hameroff S, Penrose R. Consciousness in the universe: A review of the 'Orch OR' theory. Phys Life Rev 2014;11:39-78.

70. Guillaud L, Bosc C, Fourest-Lieuvin A, et al. STOP proteins are responsible for the high degree of microtubule stabilization observed in neuronal cells. J Cell Biol 1998;142:167-79.

71. Craddock TJ, Tuszynski JA, Hameroff S. Cytoskeletal signaling: Is memory encoded in microtubule lattices by CaMKII phosphorylation? PLoS Comput Biol 
2012;8:e1002421.

72. Tuszyński JA, Hameroff S, SatariĆ MV, et al. Ferroelectric behavior in microtubule dipole lattices: Implications for information processing, signaling and assembly/ disassembly. J Theor Biol 2015;174:371-80.

73. Brunden KR, Yao Y, Potuzak JS, et al. The characterization of microtubule-stabilizing drugs as possible therapeutic agents for Alzheimer's disease and related tauopathies. Pharmacol Res 2011;63:341-51.

74. Amos LA, Baker TS. The three-dimensional structure of tubulin protofilaments. Nature 1979;279:607-12.

75. Bryan RF. Biochemistry. Second Edition by Voet D, Voet JG. Acta Crystallogr 1996;52:610.

76. Sánchez C, Díaz-Nido J, Avila J. Phosphorylation of microtubule-associated protein 2 (MAP2) and its relevance for the regulation of the neuronal cytoskeleton function. Prog Neurobiol 2000;61:133-68.

77. Hameroff SR, Penrose R. Orchestrated reduction of quantum coherence in brain microtubules. A Model Conscious Neural Netw World 1995;5:793-804.

78. Hameroff S, Nip A, Porter M, et al. Conduction pathways in microtubules, biological quantum computation, and consciousness. BioSystems 2002;64:149-68.

79. Hameroff SR, Penrose R. Conscious Events as Orchestrated Space-Time Selections. 2015.

80. Hameroff S, Penrose R. Orchestrated reduction of quantum coherence in brain microtubules: A model for consciousness. NeuroQuantology 2007;5:1-8.

81. Penrose R, Hameroff SR. What 'Gaps'? Reply to Grush and Churchland. J Conscious Stud 1995;2:98-111.

82. Hameroff S. Quantum computing in microtubules-an intra-neural correlate of consciousness? Cogn Stud 1997. doi:10.11225/jcss.4.3_67.

83. Ghosh S, Sahu S, Bandyopadhyay A. Evidence of massive global synchronization and the consciousness. Phys Life Rev 2014;11:83-4.

84. Tuszynski JA. The need for a physical basis of cognitive process. Comment on 'Consciousness in the universe: A review of the "Orch OR" theory' by Hameroff and Penrose. Phys Life Rev 2014;11:79-80.

85. Chopra D. Reality and consciousness: A view from the East. Phys Life Rev 2014;11:81-2.

86. Lucas J. The face of freedom. Comment on 'Consciousness in the universe: A review of the "Orch OR" theory' by Stuart Hameroff and Roger Penrose. Phys Life Rev 2014;11:87-8.

87. Jumper CC, Scholes GD. Life-Warm, wet and noisy?
Phys Life Rev 2014;11:85-6.

88. Pino S, Di Mauro E. How to conciliate Popper with Cartesius. Comment on: 'Consciousness in the universe. A review of the "Orch OR" theory' by S. Hameroff and R. Penrose. Phys Life Rev 2014;11:91-3.

89. Baars BJ, Edelman DB. Consciousness, biology and quantum hypotheses. Phys Life Rev 2012;9:285-94.

90. Tandy C. Are you (almost) a zombie?: Conscious thoughts about 'Consciousness in the universe' by Hameroff and Penrose. Phys Life Rev 2014;11:89-90; discussion 94-100.

91. Hameroff S. Quantum brain biology complements neuronal assembly approaches to consciousness: Comment on 'Consciousness, biology and quantum hypotheses' by Baars and Edelman. Phys Life Rev 2012;9:303-5.

92. Bernroider $\mathrm{G}$. Is there space for the quantum domain in consciousness research?. Comment on 'Consciousness, biology, and quantum hypotheses' by Baars and Edelman. Phys Life Rev 2012;9:301-2.

93. Klimesch W, Arora A. The EEG frequency architecture, coupled oscillations and consciousness. Comment on 'Consciousness, biology and quantum hypotheses' by Baars and Edelman. Phys Life Rev 2012;9:295-6.

94. Seth AK. Putting Descartes before the horse: Quantum theories of consciousness: Comment on "Consciousness, biology, and quantum hypotheses" by Baars \& Edelman. Phys Life Rev 2012;9:297-8; discussion 306-7.

95. Wessel JR. From "Neural correlates of consciousness" to "Neural causes of consciousness": A commentary on "Consciousness, biology and quantum hypotheses", by Bernard J. Baars and David E. Edelman. Phys Life Rev 2012;9:299-300; discussion 306-7.

96. Craddock TJA, Kurian P, Preto J, et al. Anesthetic Alterations of Collective Terahertz Oscillations in Tubulin Correlate with Clinical Potency: Implications for Anesthetic Action and Post-Operative Cognitive Dysfunction. Sci Rep 2017;7:9877.

97. Xi J, Liu R, Asbury GR, et al. Inhalational anestheticbinding proteins in rat neuronal membranes. $\mathrm{J}$ Biol Chem 2004;279:19628-33.

98. Pan JZ, Xi J, Tobias JW, et al. Halothane binding proteome in human brain cortex. J Proteome Res 2007;6:582-92.

99. Emerson DJ, Weiser BP, Psonis J, et al. Direct modulation of microtubule stability contributes to anthracene general anesthesia. J Am Chem Soc 2013;135:5389.

100. Craddock TJ, St George M, Freedman H, et al. Computational predictions of volatile anesthetic interactions with the microtubule cytoskeleton: 
implications for side effects of general anesthesia. PLoS One 2012;7:e37251.

101. Hameroff SR. The brain is both neurocomputer and quantum computer. Cogn Sci 2007;31:1035-45.

102. Hameroff S. Quantum walks in brain microtubules - A biomolecular basis for quantum cognition? Top Cogn Sci 2014;6:91-7.

103. Tonello L, Cocchi M, Gabrielli F, et al. On the possible quantum role of serotonin in consciousness. J Integr

Cite this article as: $\mathrm{Li} \mathrm{T}$, Tang $\mathrm{H}$, Zhu J, Zhang JH. The finer scale of consciousness: quantum theory. Ann Transl Med 2019;7(20):585. doi: 10.21037/atm.2019.09.09
Neurosci 2015;14:295-308.

104. Craddock TJ, Priel A, Tuszynski JA. Keeping time: Could quantum beating in microtubules be the basis for the neural synchrony related to consciousness? J Integr Neurosci 2014;13:293-311.

105.Li N, Lu D, Yang L, et al. Nuclear Spin Attenuates the Anesthetic Potency of Xenon Isotopes in Mice: Implications for the Mechanisms of Anesthesia and Consciousness. Anesthesiology 2018;129:271-277. 